\title{
Immunopotentiator Aikejia improves the therapeutic efficacy of PD-1/PD-L1 immunosuppressive pathway in CT26.WT cancer cell
}

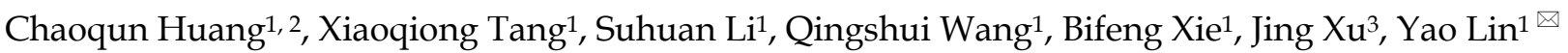 \\ 1. Key Laboratory of Optoelectronic Science and Technology for Medicine of Ministry of Education, College of Life Sciences, Fujian Normal University, \\ Fuzhou 350117, Fujian Province, People's Republic of China. \\ 2. Central laboratory, The Fifth Hospital of Xiamen, Xiamen 361101, Fujian Province, People's Republic of China. \\ 3. Shanhe Pharmaceutical Co., Ltd., No789, Hangang West Road, Chigang Overseas Chinese Economic Development Zone, Putian 351100, Fujian Province, \\ People's Republic of China. \\ $\triangle$ Corresponding author: Dr. Yao Lin, College of Life Sciences, Qishan Campus, Fujian Normal University, Fuzhou, Fujian Province, P.R. China, 350117. Email: \\ yaolin@fjnu.edu.cn; yaolinfjfz@gmail.com; Tel: +86-(0)591-22860592; Fax: +86-(0)591-22860592.
}

(C) Ivyspring International Publisher. This is an open access article distributed under the terms of the Creative Commons Attribution (CC BY-NC) license (https://creativecommons.org/licenses/by-nc/4.0/). See http://ivyspring.com/terms for full terms and conditions.

Received: 2018.09.03; Accepted: 2019.05.15; Published: 2019.06.09

\begin{abstract}
Tumors can escape immunosurveillance through immunocheckpoint such as the PD-1/PD-L1 pathway. Aikejia comes from Nocardia rubra cell-wall skeleton and can increase the number of inflammatory factors and immune cells. In this work, we showed that the levels of PD-LI increase in CT26.WT xenograft after subcutaneous injection of Aikejia in mice, but Aikejia did not induce the expression of PD-L1 in vitro. When we treated the mice with Aikejia and blocked PD-1/PD-LI pathway in vivo at the same time, the CT26.WT xenografts were significantly inhibited or eliminated, which was better than single treatment alone. Our results suggested that Aikejia may be an effective adjuvant for PD-1/PD-L1 immunotherapy.
\end{abstract}

Key words: PD-L1, Aikejia, immunotherapy

\section{Introduction}

Immunoinhibitory receptor programmed death-1 (PD-1/PDCD1) is widely expressed on the membrane surface of activated $\mathrm{T}$ cells, $\mathrm{B}$ cells, and myeloid cells. PD-1 is related to the regulation of peripheral tolerance and immune response [1-3]. Programmed death-1 ligand-1 (PD-L1, also named CD274 or B7-H1), has been identified as the ligand for PD-1. A large number of studies have confirmed that PD-L1 is highly expressed on the surface of most tumor cells in patients [4-7]. When PD-1 binds specifically with PD-L1, they form an inhibitory complex to block the activity of immune cells. This allows the tumors to escape the surveillance of the immune system [8-11]. The antibodies developed for blocking this inhibitory pathway have been used clinically and displayed promising therapeutic effect.

However, some patients are unable to respond effectively to these inhibitory antibodies [12, 13]. The reason for this may be that the immunogenicity of most patients with spontaneous tumors is weak. There are only few immune cells or infiltrating $\mathrm{T}$ cells in the tumor microenvironment, resulting in insufficient immunosuppressive effect on the tumor $[14,15]$. In view of the above problems, we investigated whether Aikejia can be used as a potential immune adjuvant for PD-1/PD-L1 antibody therapy in an animal model.

Aikejia is an immunomodulator drug obtained through a series of chemical extraction and refining after the Nocardia rubra was broken [16]. It can induce the production of endogenous interferon (IFN) and tumor necrosis factor (TNF) and improve the immune activity of $\mathrm{T}$ cells, macrophages and NK cells [17-21]. Aikejia has been approved to control pleural effusion and ascites caused by various tumors [19]. It can also be used as an adjuvant to treat lung cancer, malignant melanoma, bladder cancer, malignant lymphoma, advanced gastric cancer and esophageal cancer [22, 23]. 
In this study, we used a stable inducible knockdown system to mimic the blockade of PD-1/PD-L1 interaction. At the same time, mice were injected subcutaneously with Aikejia. The efficacy of the combination of the PD-L1 inhibition and Aikejia was then evaluated.

\section{Materials and Methods}

\section{Antibodies and chemicals}

GAPDH (2118) antibody was purchased from Cell Signaling Technology (Boston, USA). B7-H1/PD-L1 (AF1019) antibody was purchased from R\&D Systems (Minnesota, USA). Two-step assay kit of Goat (PV-9003) was purchased from ZSGB-BIO (Beijing, China). IRDye 800CW Donkey anti-Goat (P/N 925-32214) antibody and IRDye 680RD Goat-anti-Rabbit (P/N 926-68071) antibody were purchased from LI-COR (Nebraska, USA).

Doxycycline (D9891) was purchased from Sigma-Aldrich (St Louis, USA) and used at $15 \mathrm{mg} / \mathrm{mL}$ in in vivo experiment or $200 \mathrm{ng} / \mathrm{mL}$ in in vitro experiment. Recombinant Murine IFN- $\gamma$ (315-05) was purchased from Peprotech (Rocky Hill, NJ, USA) and used at $25 \mathrm{ng} / \mathrm{mL}, 50 \mathrm{ng} / \mathrm{mL}$ or $100 \mathrm{ng} / \mathrm{mL}$ respectively in cell culture experiments. Puromycin (A11138-03) was purchased from Thermo Fisher Scientific (Massachusetts, USA) and used at $8 \mu \mathrm{g} / \mathrm{mL}$. Aikejia was provided by Shanhe Pharmaceuticals (Putian, China), dissolved in $100 \mu \mathrm{L}$ PBS and used at $0.5 \mu \mathrm{g}, 1 \mu \mathrm{g}$ or $2 \mu \mathrm{g}$ per mouse. Matrigel (356234) was purchased from Corning (New York, USA).

\section{Cloning procedures}

Five PD-L1-specific shRNA (shRNA1, shRNA2, shRNA3, shRNA4, shRNA5) and a scrambled control (Table 1) from Sigma (St Louis, USA) was cloned into a Tet-pLKO-puro backbone purchased from Addgene (Cambridge, UK) respectively. The plasmids were mixed with XhoI (Thermo Fisher Scientific, Massachusetts, USA) at $37^{\circ} \mathrm{C}$ for $15 \mathrm{~min}$. Cleaved DNA fragments were separated on $2 \%$ agarose gels to identify successful cloning. These verified plasmids were transfected into packaging cells and then virus-containing medium supernatants were used to infect CT26.WT tumor cells using polybrene methodology. For conditional knockdown, stable cell lines were generated after screening with $8 \mu \mathrm{g} / \mathrm{mL}$ puro for three weeks. The expanded surviving cells were treated with $200 \mathrm{ng} / \mathrm{mL}$ Dox and $100 \mathrm{ng} / \mathrm{mL}$ IFN- $\gamma$ for $48 \mathrm{~h}$. The efficiency of PD-L1 knockdown was detected by western blot.

\section{Animals and Cell culture}

Adult female Balb/c mice were purchased from Slaccas (Shanghai, China). All mice were identically raised and housed in rearing cage in compliance with The Guide for Care and Use of Laboratory Animals (National Research Council). This study was performed with the approval of the ethics committee of Fujian Normal University. The 4T1, CT26.WT and RAG cells were obtained from the Cell Line Bank, Chinese Academy of Sciences. All the cells were verified by STR genotyping and tested negative for mycoplasma. Both 4T1 and CT26.WT were grown in RPMI 1640 medium (Biological Industries, CROMWELL, USA), whereas RAG was maintained in MEM medium (Biological Industries) with $1 x$ Non-Essential Amino Acids Solution (Thermo Fisher Scientific, Massachusetts, USA). All cultures were supplemented with $10 \%$ fetal bovine serum (Biological Industries, CROMWELL, USA), 100 units/mL penicillin $\mathrm{G}$ and $100 \mu \mathrm{g} / \mathrm{mL}$ streptomycin (BBI Life Sciences Corporation, Shanghai, China). All cells were grown at $37^{\circ} \mathrm{C}$ in a humidified incubator containing $5 \% \mathrm{CO}_{2}$.

Table 1. Primer sequences used for the construction of stable cell lines

\begin{tabular}{lll}
\hline name & sequence & knockdown \\
\hline scr & 5'CCGGTTCTCCGAACGTGTCACGTCTCGAGACGTGACA & / \\
& CGTTCGGAGAATTTT 3' & \\
sh1 & 5'CCGGCCGAAATGATACACAATTCGACTCGAGTCGAAT & $81 \%$ \\
& TGTGTATCATTTCGGTTTT 3' & \\
sh2 & 5'CCGGGCCACTTCTGAGCATGAACTACTCGAGTAGTTC & $90 \%$ \\
& ATGCTCAGAAGTGGCTTTT 3' & \\
sh3 & 5'CCGGGCGTTGAAGATACAAGCTCAACTCGAGTTGAGC & / \\
& TTGTATCTTCAACGCTTTT 3' & \\
sh4 & 5'CCGGGTTTACTATCACGGCTCCAAACTCGAGTTTGGA & 21\% \\
& GCCGTGATAGTAAACTTTTT 3' & \\
sh5 & 5'CCGGCAGGCGTTTACTGCTGCATAACTCGAGTTATGC & $/$ \\
& AGCAGTAAACGCCTGTTTT 3' & \\
\hline
\end{tabular}

\section{Western blot}

For tumor cells, after discarding the cell culture media, the remaining cells were washed with PBS. All the remaining liquid was removed. 100 200 $\mu \mathrm{L}$ ice-cold RIPA buffer $(150 \mathrm{mM} \mathrm{NaCl}, 0.5 \%$ sodium deoxycholate, $1 \%$ NP-40, $50 \mathrm{mM}$ Tris $\mathrm{HCl} \mathrm{pH} 8.0$, $0.1 \%$ SDS) containing protease inhibitors (Roche, Basel, Switzerland) was then added to the cell culture dishes. Cells were collected with cell scrapers and then placed on ice for $20 \mathrm{~min}$. For tumor tissue, tissue was weighed $(25 \mathrm{mg})$ before addition of lysis buffer at 1 to 10 ratio (mg: $\mu \mathrm{L})$. The tumor tissues were fully grinded with a motor-driven tissue grinder and then placed on ice for $20 \mathrm{~min}$. The supernatant was collected after centrifugation for $10 \mathrm{~min}$ at $4^{\circ} \mathrm{C}, 13000$ rpm. Protein concentration was measured by Pierce BCA Protein Assay Kit (Thermo Fisher Scientific, Massachusetts, USA). Protein samples $(60 \mu \mathrm{g})$ were separated by SDS-PAGE, transferred to nitrocellulose membrane (GE Healthcare Life Sciences, Boston, USA) and then western blotting was performed 
following standard protocols. Finally, the blots were imaged and quantified using the Odyssey Infrared Imaging System (LI-COR, Nebraska, USA).

\section{Tumor growth studies}

The 4T1, CT26.WT and RAG tumor cells (1.5 x $\left.10^{6}\right)$ were injected subcutaneously with a volume of $100 \mu \mathrm{L}$ (including half the volume of matrigel) into 4-6-week-old female Balb/C mice. After a week, mice were randomized and treatment was initiated wh en the average tumor volume was $150-200 \mathrm{~mm}^{3}$. The volumes of the tumors were measured once every other day with a vernier caliper, and the tumor volumes were calculated as: Volume $=0.5 \times(\text { width })^{2}$ $x$ (length).

\section{In vitro growth inhibition assay}

CT-26.WT cells in the logarithmic growth phase were seeded into 96-well plates at concentrations of 10000 cells/well. After $24 \mathrm{~h}$, the cells were respectively cultured in $100 \mu \mathrm{L}$ complete RPMI 1640 medium in the presence of $0,6.25,12.5,25$ and 50 $\mu \mathrm{g} / \mathrm{mL}$ Aikejia for $48 \mathrm{~h}$. After treatment, $10 \mu \mathrm{L}$ CCK8 (TransGen Biotech, Beijing, China) was added into each well, and the plates were incubated for $1 \mathrm{~h}$ at $37^{\circ} \mathrm{C}$. Then, the absorbance was measured at $450 \mathrm{~nm}$ using Synergy HT Multi-Mode Microplate Reader (BioTek, Winooski, VT, USA). Statistical results were obtained from three independent experiments.

\section{Immunohistochemistry (IHC)}

Briefly, tumor tissues were stripped from the mice, fixed in $4 \%$ formaldehyde, embedded in paraffin and sectioned. Sections were dehydrated by gradient alcohol, cleared with xylene, repaired antigen with citrate buffer solution under high pressure condition, and then the primary and secondary antibodies were added respectively for staining. PD-L1 staining was counterstained with hematoxylin. Above experimental procedures and conditions were performed according to the instructions for immunohistochemical kit purchased from ZSGB-BIO (Beijing, China). At least 3 tumors per experimental condition and at least 3 random fields per section were imaged. Pictures were taken with 10-40x objectives.

\section{Statistics}

Graphs were presented as the mean \pm SEM. One asterisk $\left(^{*}\right)$ represented $\mathrm{P}<0.05$ and indicated statistically significant. Two asterisks ${ }^{* *}$ ) represented $P<0.01$. Differences between groups were analyzed using one-way ANOVA or Student's t-test. Analyses were performed with Prism 5.0 (GraphPad Software Inc., La Jolla, CA, USA).

\section{Results}

Currently none of the PD-L1 inhibitory antibodies have entered Chinese market. Therefore, in order to simulate their inhibitory effect, we decided to use a Tet-on system to construct a stable cell lines in which the PD-L1 protein expression can be inducibly knocked down upon Dox induction.

We first set out to select mouse cancer cell lines that are ideal for this project. The 4T1, CT26.WT and RAG tumor cells from Balb/C mice were treated with different concentrations of murine IFN-y in vitro. The PD-L1 expression was significantly induced by IFN- $\gamma$ in all three cells in a dose dependent manner (Figure $1 A \& 1 B)$. Next, we inoculated these three cells to the back and armpits of female Balb/C mice respectively. The 4T1 and CT26.WT cells successfully formed xenografts in vivo (Figure 1C). However, equal amount of RAG cells failed to form xenografts. Moreover, both IHC and western blot (Figure 1D-F) showed that the PD-L1 protein expression was induced in 4T1 and CT26.WT xenografts. The staining of secondary antibody only controls were showed on the right of IHC (Figure 1D), which confirmed the specific staining of PD-L1. Taking into account the tumor formation rate and the expression level of PD-L1, CT26.WT cell was selected for the follow-up experiments and stable cell lines were successfully constructed. Of all the shRNAs (Figure 2A), Sh2 displayed the strongest suppressive effect (Figure 2B\&2C). Therefore, we selected sh2 to verify the knockdown effect in vivo. In the CT26.WT xenograft, upon Dox induction, the average knockdown effect of PD-L1 detected by western blot is about $53.7 \%$ (Figure 2D\&2E).

Before we carried out the combinational treatment, we treated tumor-bearing mice with three different concentrations of Aikejia in order to select a suitable dose (Figure 3A). The mice were randomized and treatment was initiated when the average tumor volume reached $150-200 \mathrm{~mm}^{3}$. The tumors began to be significantly inhibited when the mice were treated with $1.0 \mu \mathrm{g}$ Aikejia compared to the control group. However, the inhibition rate did not increase significantly when the mice were treated with $2 \mu \mathrm{g}$ compared with $1 \mu \mathrm{g}$ Aikejia. (Figure 3B\&3C). The protein levels of PD-L1 in CT26.WT xenografts was up-regulated with increasing amount of Aikejia when examined with IHC (Figure 3D) or western blot (Figure 3E\&3F). Based on the above results, 1ug Aikejia is a sufficient for the follow-up experiments. 
A

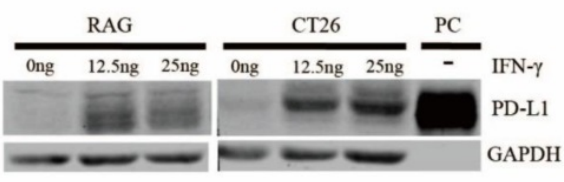

B

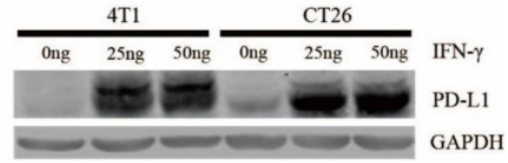

C

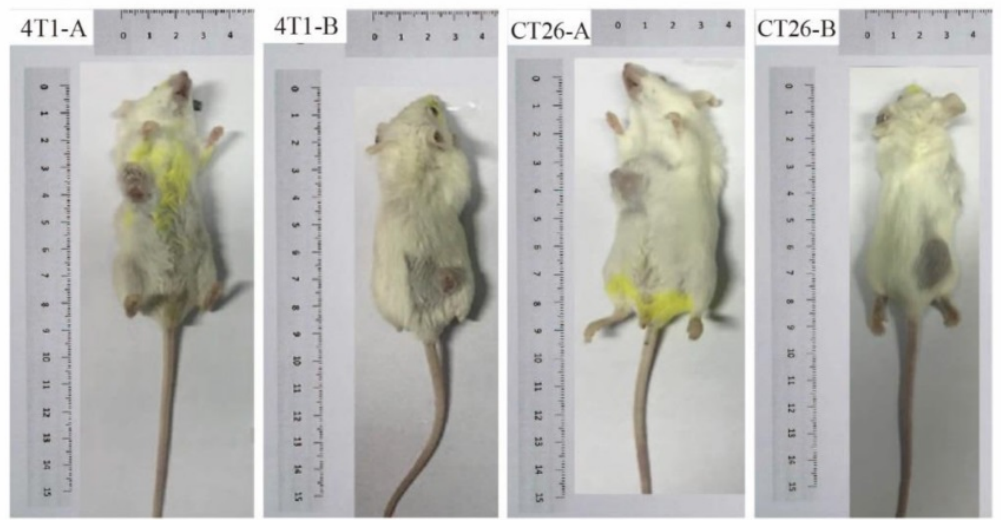

D

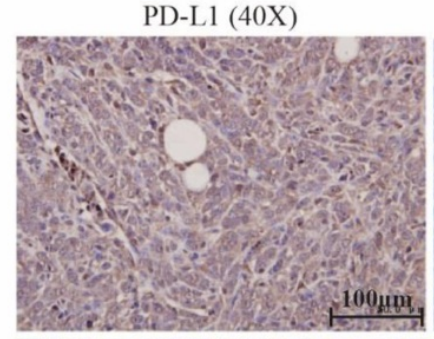

only second antibody

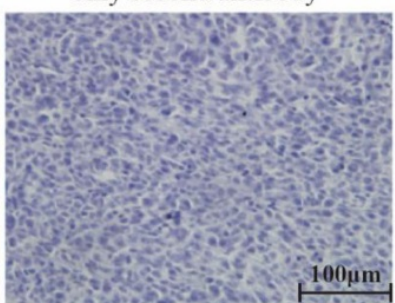

$4 \mathrm{~T} 1$
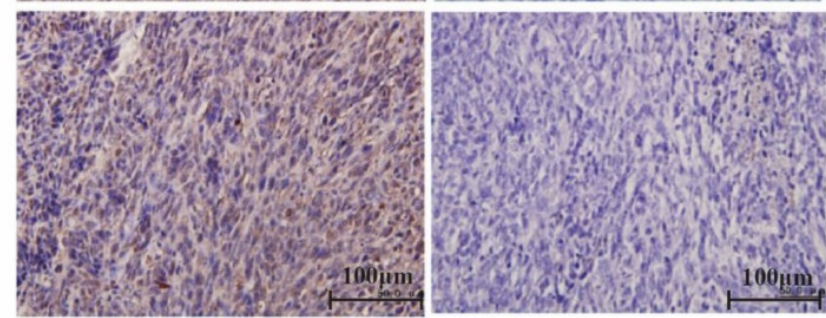

E

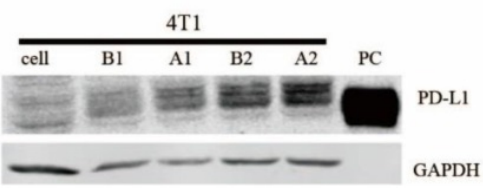

F

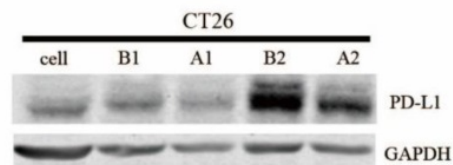

Figure 1. Detection of PD-LI expression in murine tumor cells in vitro and vivo. (A) CT26.WT and RAG were treated with different concentrations of IFN- $\mathrm{Y}$ ( $0,12.5$ $\mathrm{ng} / \mathrm{mL}, 25 \mathrm{ng} / \mathrm{mL}$ ) for $48 \mathrm{~h}$, the expression level of PD-L1 was detected by western blot. PC is purified Murine PD-L1 protein (15 ng) and used as a positive control. The experiments were repeated three times. (B) $4 \mathrm{Tl}$ and CT26.WT cells were treated with different concentrations of IFN-Y (0, $25 \mathrm{ng} / \mathrm{mL}$, $50 \mathrm{ng} / \mathrm{mL})$ for $48 \mathrm{~h}$, the expression level of PD-L1 was detected by western blot. (C) CT26.WT and 4T1 cells $\left(1.5 \times 10^{6}\right)$ were injected subcutaneously into the back and armpits of 4-6-week-old female Balb/C mice respectively. The tumor formation rate was observed after two weeks. -A: tumor on armpit; -B: tumor on back. (D) The xenografts of 4T1 and CT26.WT were obtained 1 week later. Expression level of PD-L1 in xenografts was detected by immunohistochemistry (Secondary antibody only controls were shown on the right). The antibody dilution ratio was 1:200. Images are representative of 3 distinct tumors and 3 images per tumor. Scale bar: $100 \mu \mathrm{m}$. (E\&F) Expression level of PD-L1 in xenografts of 4T1 and CT26.WT was detected by western blot. $\mathrm{A} 1$ and $\mathrm{A} 2$ : armpits xenografts from different mice; $\mathrm{B} 1$ and $\mathrm{B} 2$ : back xenografts from different mice.

Next, we started a combination experiment when the xenograft size grew to $300 \mathrm{~mm}^{3}$. Each mouse was injected subcutaneously with the stable cells containing scramble shRNA on the left back and shRNA2 on the right (Figure 4A). Blockade of the PD-1/PD-L1 pathway had a similar inhibitory effect as Aikejia alone. When PD-L1 was inducibly knocked down by Dox and Aikejia was used, the tumors were significantly suppressed and almost disappeared (Figure 4B-4D). The PD-L1 knockdown effect was further confirmed using IHC. From the staining of the tissue sections, it can be observed that the brownness of the tissue and the proportion of brown area were reduced (Figure $4 \mathrm{E}$ ). These results suggested that there may be a synergistic effect of the combination of Aikejia and the blockade of PD-1/PD-L1 interaction. 
Later, we verified that Aikejia did not efficiently suppress CT26.WT tumor cells in vitro. When the dose of Aikejia reached $50 \mu \mathrm{g} / \mathrm{mL}$ in medium, the inhibition rate was only $10.2 \%$ for CT26.WT cells (Figure 5A). It was much higher than the amount of $1 \mu \mathrm{g}$ per mouse in vivo, suggesting that Aikejia may exert its effect on tumor cells indirectly. In addition, different doses of Aikejia failed to induce PD-L1 protein expression in CT26.WT cells in vitro. However, small amount of INF-y $(50 \mathrm{ng} / \mathrm{mL})$ can induce the expression of PD-L1 protein (Figure 5B), suggesting Aikejia cannot directly activate the immunosurveillance system in cancer cells.

\section{Discussion}

PD-1 is an inhibitory receptor for T cells, which tumors utilize to escape from immune suppression. Our results showed that Aikejia can up-regulate the expression of PD-L1 in tumor tissues. However, it can inhibit tumor cells in vivo. These seemingly contradictory results may be explained by a dynamic balance model. Aikejia may increase the release of inflammatory factors in vivo. High concentration of inflammatory factors may kill the tumor ${ }^{[24-25]}$ and induce elevated expression of PD-L1 on tumor surface, allowing part of the tumor to evade the killing of the immune system. Therefore, when Aikejia was combined with the blockade of the PD-1/PD-L1 pathway, the tumor suppressive effect may be multiplied. More experiments need to be done to further investigate and verify the underlying mechanism of our observation.

In our animal model, we induced the knockdown of PD-L1 through the Tet-on system. It reduces the expression of PD-L1 protein from the inside of the cell, and there is no spatial blockage on the cell membrane, which is different from the clinical applied antibodies. In the absence of steric hindrance, $\mathrm{T}$ cells may be closer to the tumor cells, making our model may be more significant than blockade treatment with PD-1/PD-L1 antibodies. It will be ideal if the combinational efficacy can be verified using Aikejia and drugs such as Keytruda or Opdivo.

A

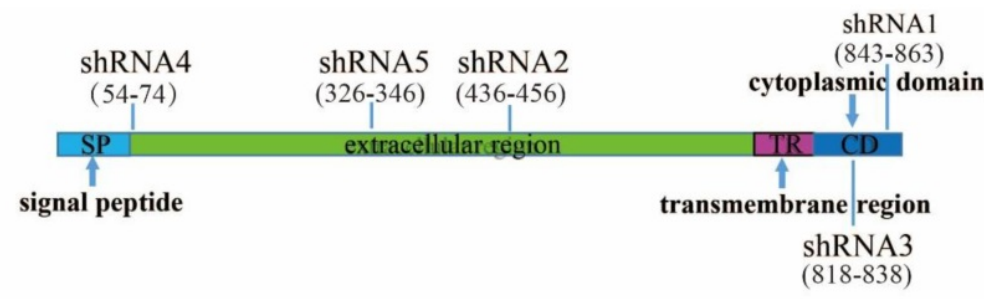

B

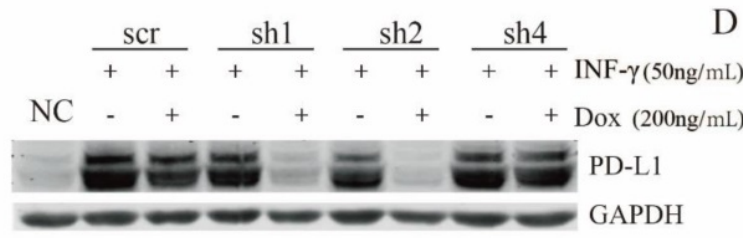

$\mathrm{D}$

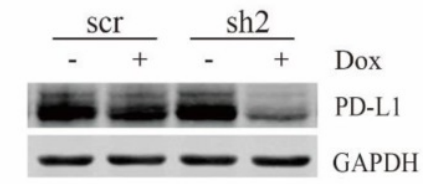

$\mathrm{C}$

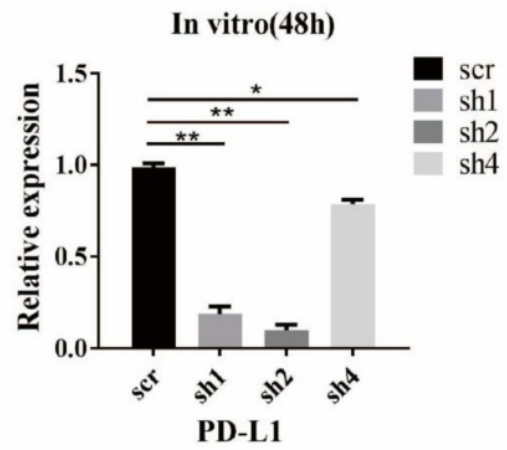

In vivo(4d)

$\mathrm{E}$

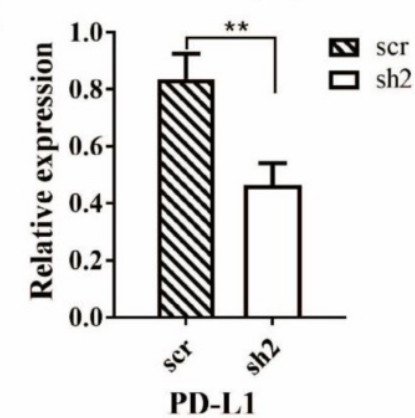

Figure 2. Verification of the PD-LI knockdown effect in the stable inducible CT26.WT cell line in vitro and vivo. (A) The schematic diagram of PD-L1 shRNA at corresponding target sites on mRNA. SP: signal peptide; ER: extracellular region; TR: transmembrane region; CD: cytoplasmic domain. (B) The stable CT26.WT cells (scramble, shRNA1, shRNA2, shRNA4) were treated with $50 \mathrm{ng} / \mathrm{mL} \mathrm{IFN-y} \mathrm{and} 200 \mathrm{ng} / \mathrm{mL}$ Dox for $48 \mathrm{~h}$ in vitro. After protein extraction, the expression level of PD-L1 was detected by western blot $(n=3)$. The quantification of the results $(B)$ was presented in $(C)$. (D\&E) The mice injected with stable CT26 tumor cells $(\mathrm{scr}$ or sh2) were treated with $3 \mathrm{mg} / \mathrm{mL}$ Dox per day. Tumors were harvested after 4 days. The expression level of PD-L1 in stable CT26.WT xenografts was detected by western blot ( $\mathrm{n}=3$ ) and Means \pm SEM are shown. The quantification of the results (D) were presented in $(E)$. 
A
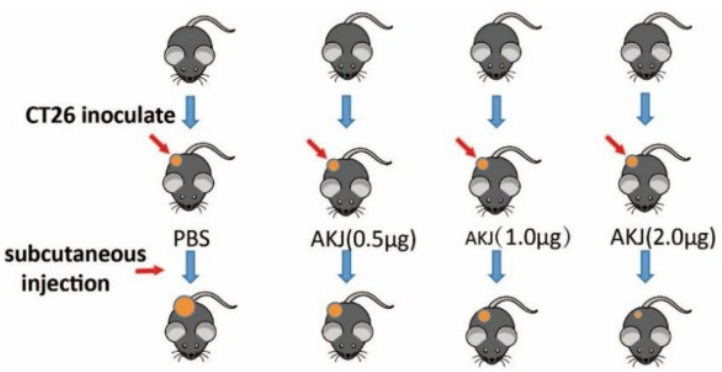

B

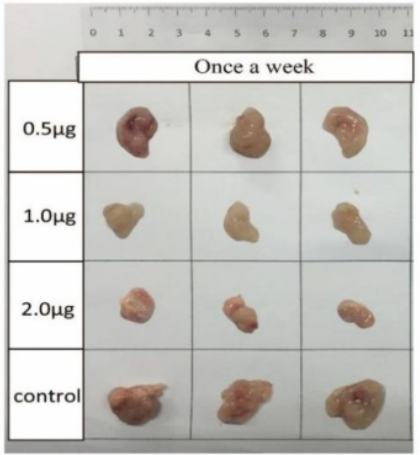

C

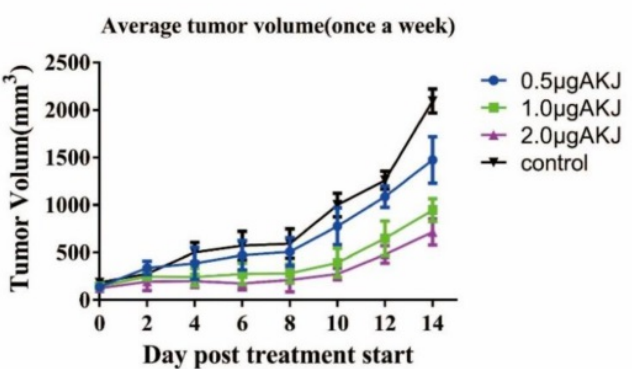

D
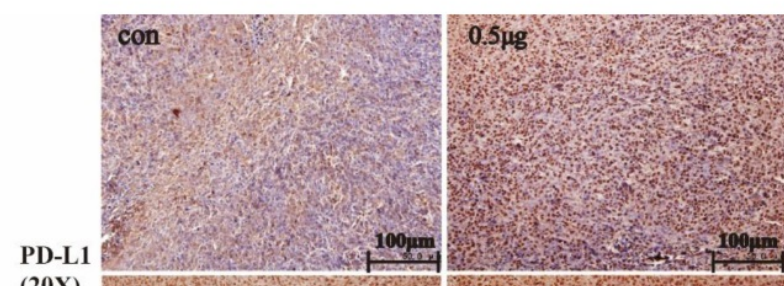

(20X)
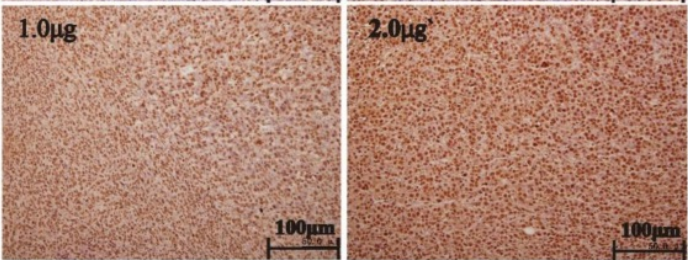

$\mathbf{E}$

F
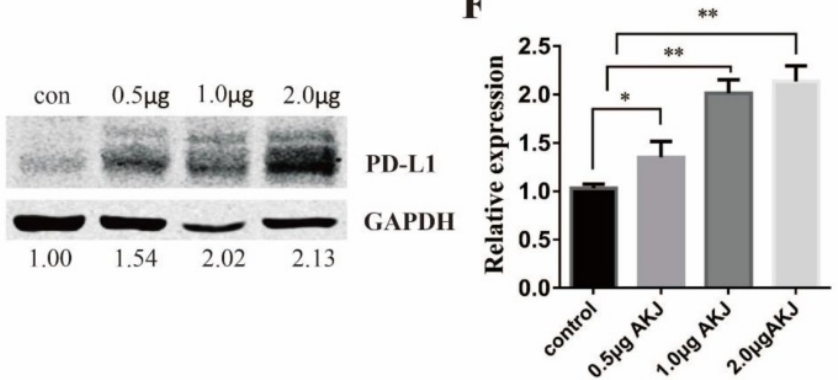

control

$0.5 \mu \mathrm{g} \mathrm{AKJ}$

$1.0 \mu \mathrm{g}$ AKJ

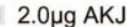

PD-L1

Figure 3. Evaluation of the effect of Aikejia on CT26 xenografts. (A) The schematic diagram of the experimental groups: tumor-bearing mice were treated with Aikejia-AKJ $(0.5,1.0,2.0 \mu \mathrm{g} /$ per mouse) once a week. (B) The CT26.WT xenograft growth of representative mice was shown after the injection of Aikejia once a week for two weeks. (C) The diameters of CT26.WT xenografts were measured by calipers and plotted as the mean \pm SEM. $n=5$ mice per condition. (D)The tumor tissue sections were obtained after two weeks. Then, the expression level of PD-L1 in mice xenografts were detected by immunohistochemistry. Images were representative of 3 distinct tumors and 3 images per tumor. Scale bar: $100 \mu \mathrm{m}$. (E) After extraction of the tumor tissues protein, the expression level of PD-L1 in mice xenografts was detected by western blot. The quantification of results was presented in $(F)$. Graphs were presented as the mean \pm SEM ( $n=3$ distinct tumors per condition).

Aikejia has been used clinically for many years and has high safety and controllability. As immunopotentiator, Aikejia may be useful for pre-treatment of cancer patients, allowing activation of the immune system before PD-1/PD-L1 antibody therapy. Our experimental results can be used as a reference for the clinical trials of Aikejia and PD-1/PD-L1 antibodies combination treatment in the future. 
A

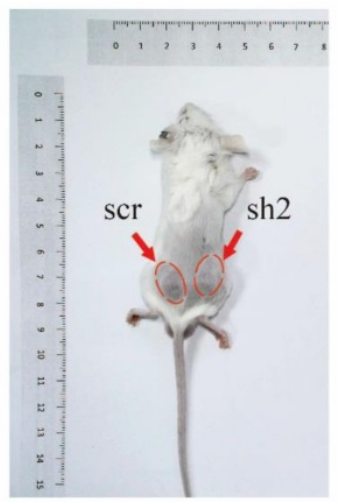

C

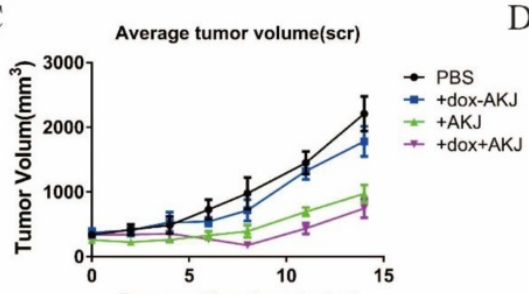

Day post treatment start
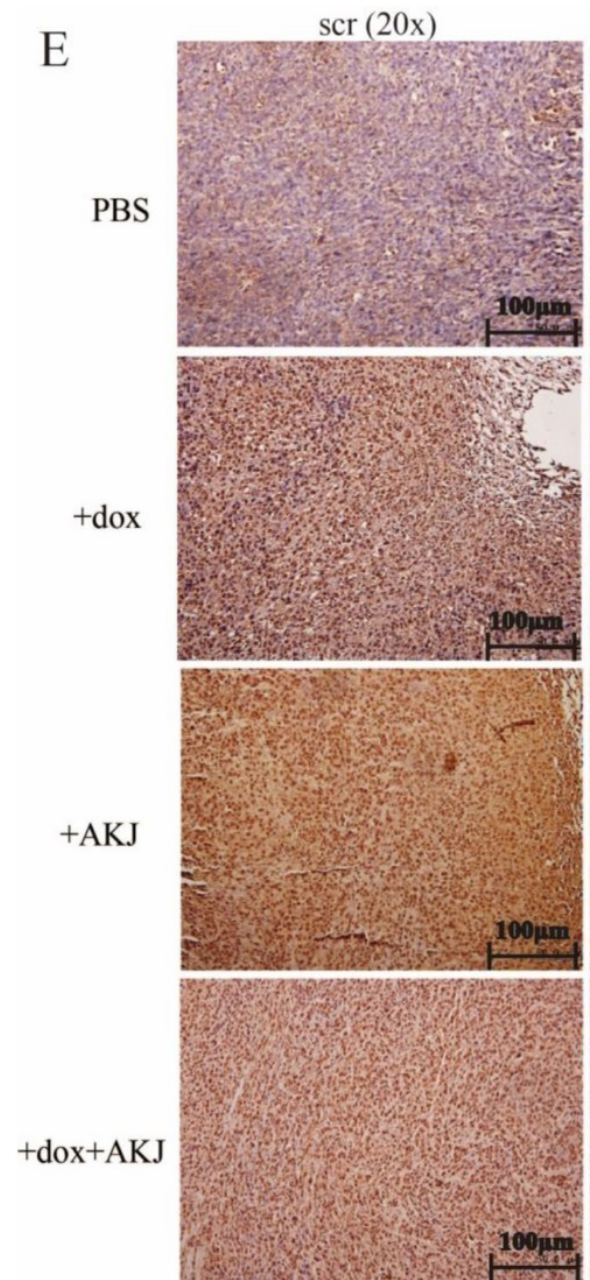

B

D
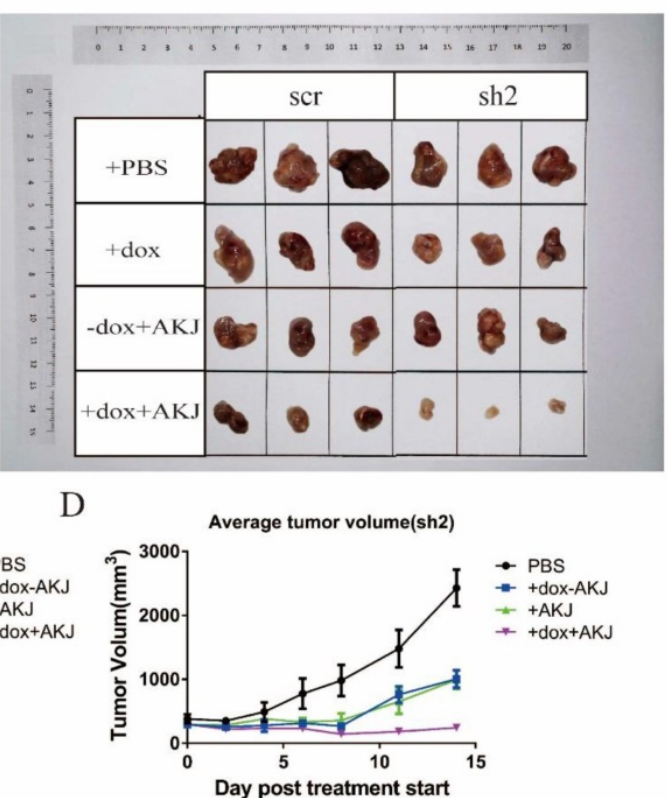

$\operatorname{sh} 2(20 x)$
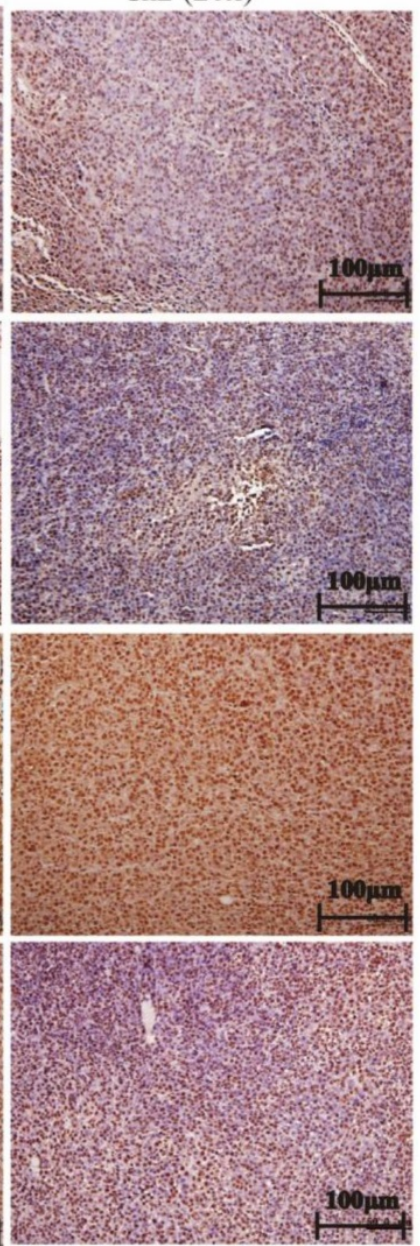

Figure 4. Evaluation of the effect of Aikejia in combination with the blockade of PD-1/PD-L1 pathway. (A) The schematic diagram of tumor inoculation: stable CT26.WT cells containing scramble shRNA (scr) and shRNA2 (sh2) were injected subcutaneously on the left and right of back respectively. (B-D) Tumor-bearing mice were treated with $100 \mu \mathrm{L}$ Aikejia $(1 \mu \mathrm{g})$ or $100 \mu \mathrm{L}$ PBS once a week and treated with or without $3 \mathrm{mg} / \mathrm{mL}$ Dox every day. The tumors were obtained two weeks later. All tumor diameters (scr and sh2) were measured by calipers and plotted as the mean \pm SEM. $n=5$ mice per condition. The representative images were presented. (E) The PD-LI expression level of CT26.WT xenografts treated with or without Aikejia in each experimental group were detected by immunohistochemistry. Images were representative of 3 distinct tumors and 3 images per tumor. Scale bar: $100 \mu \mathrm{m}$. 
A

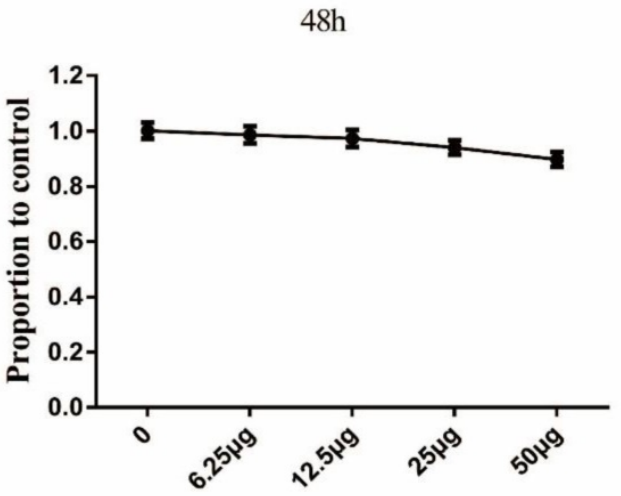

AKJ
B

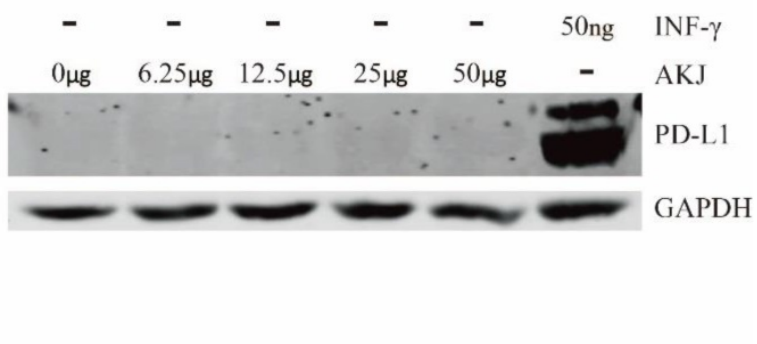

Figure 5. Evaluation of the effect of Aikejia on cell proliferation and PD-L1 expression in CT26.WT cells. (A) CT26.WT cells were treated with different doses of Aikejia $(0,6.25,12.5,25,50 \mu \mathrm{g} / \mathrm{mL})$ for $48 \mathrm{~h}$, and then the growth inhibition rate was detected by CCK 8 and Means \pm SEM were shown ( $\mathrm{n}=3$ ) (B) The PD-L1 expression level of CT26.WT cells treated with different doses of Aikejia and INF- $\gamma$ was detected by western blot. The experiments were repeated three times.

\section{Acknowledgements}

This work was supported by the International S\&T Cooperation Program of China (2016YFE0121900), the Scientific Research Innovation Team Construction Program of Fujian Normal University (IRTL1702), the United Fujian Provincial Health and Education Project for Tackling the Key Research (WKJ2016-2-27) and the Natural Science Foundation of Fujian Province (2016Y0029).

\section{Author contributions}

Chaoqun Huang designed and performed the experiments, wrote the paper and prepared figures and/or tables.

Xiaoqiong Tang, Suhuan Li and Qingshui Wang performed the experiments and analyzed the data.

Bifeng $X i e$ and Jing $\mathrm{Xu}$ contributed reagents/analysis tools.

Yao Lin conceived and designed the experiments, wrote the paper and prepared figures and/or tables.

\section{Competing Interests}

The authors have declared that no competing interest exists.

\section{References}

1. Topalian SL, Hodi FS, Brahmer JR, Gettinger SN, Smith DC, McDermott DF, et al. Safety, activity, and immune correlates of anti-PD-1 antibody in cancer. New England Journal of Medicine. 2012; 366: 2443-54

2. Baumeister SH, Freeman GJ, Dranoff G, Sharpe AH. Coinhibitory Pathways in Immunotherapy for Cancer. Annual review of immunology. 2016; 34: 539-73.

3. Bartosinska J, Zakrzewska E, Raczkiewicz D, Purkot J, Michalak-Stoma A, Kowal M, et al. Suppressed Programmed Death 1 Expression on CD4(+) and CD8(+) T Cells in Psoriatic Patients. Mediators of inflammation. 2017; 2017: $1-8$.

4. Curiel TJ, Wei S, Dong H, Alvarez X, Cheng P, Mottram P, et al. Blockade of B7-H1 improves myeloid dendritic cell-mediated antitumor immunity. Nature Medicine. 2003; 9: 562-7.

5. Akbari $O$, Stock $P$, Singh AK, Lombardi V, Lee WL, Freeman GJ, et al. PD-L1 and PD-L2 modulate airway inflammation and iNKT-cell-dependent airway hyperreactivity in opposing directions. Mucosal immunology. 2010; 3: 81-91.
6. Reck M RD, Robinson A G, et al. . Pembrolizumab versus docetaxel for previously treated, PD-L1-positive, advanced non-small-cell lung cancer (KEYNOTE-010): a randomised controlled trial. Lancet. 2016; 387: 1540-50.

7. Reck M, Rodriguez-Abreu D, Robinson AG, Hui R, Csoszi T, Fulop A, et al. Pembrolizumab versus Chemotherapy for PD-L1-Positive Non-Small-Cell Lung Cancer. The New England journal of medicine. 2016; 375: 1823-33.

8. Chen L, Zhang Z, Chen W, Zhang Z, Li Y, Shi M, et al. B7-H1 up-regulation on myeloid dendritic cells significantly suppresses $T$ cell immune function in patients with chronic hepatitis B. Journal of Immunology. 2007; 178: 6634-41.

9. Nishino M, Giobbiehurder A, Gargano M, Suda M, Ramaiya NH, Hodi FS. Developing a common language for tumor response to immunotherapy: immune-related response criteria using unidimensional measurements. Clinical Cancer Research An Official Journal of the American Association for Cancer Research. 2013; 19: 3936-43.

10. Chi YO, Young KH. Checkpoint inhibitors in hematological malignancies. Journal of Hematology \& Oncology. 2017; 10: 103-18.

11. Goodman A, Patel SP, Kurzrock R. PD-1-PD-L1 immune-checkpoint blockade in B-cell lymphomas. Nature Reviews Clinical Oncology. 2017; 14: 203-20.

12. Pennock GK, Waterfield W, Wolchok JD. Patient responses to ipilimumab, a novel immunopotentiator for metastatic melanoma: how different are these from conventional treatment responses? American Journal of Clinical Oncology. 2012; 35: 606-11.

13. Larkin J, Chiarionsileni V, Gonzalez R, Grob JJ, Cowey CL, Lao CD, et al. Combined Nivolumab and Ipilimumab or Monotherapy in Previously Untreated Melanoma. New England Journal of Medicine. 2015; 373: 23-34.

14. Sharma P, Allison JP. The future of immune checkpoint therapy. Science. 2015; 348: 56-61.

15. Hamid O, Robert C, Daud A, Hodi FS, Hwu WJ, Kefford R, et al. Safety and tumor responses with lambrolizumab (anti-PD-1) in melanoma. New England Journal of Medicine. 2013: 369: 134-44.

16. Rao GQ, Pan MP, Xie BF. Isolation and Identification of N-CWS-PG and Experimental Study on Tumor Suppression. Pharmaceutical Biotechnology. 2016

17. Yokota S, Shirasaka T, Nishikawa H, Hosoe S, Ikeda T, Komuta K, et al. Augmentative effect of Nocardia rubra cell-wall skeleton (N-CWS) on lymphokine-activated killer (LAK) cell induction. Cancer Immunology Immunotherapy. 1988; 26: 11-7.

18. Shirasaka T, Kawase I, Okada M, Kitahara M, Ikeda T, Komuta K, et al. Augmentative effect of Nocardia rubra cell-wall skeleton on the induction of human lymphokine-activated killer (LAK) cells by the production of LAK cell helper factor(s). Cancer Immunol Immunother. 1989; 30: 195-204.

19. Zhang ZL, Tang WL, Yang HJ, Wang J. Effect of Nocardia rubra Cell Wall Skeleton(N-CWS) on Transplanted Tumor and Immunological Function in Mice Given Through Oral Administration. Natural Product Research \& Development. 2010; 22: 228-31.

20. Wang G, Jie W, Miao M, Dou H, Ning N, Shi $M$, et al. Nocardia rubra Cell-Wall Skeleton promotes CD4 + T cell activation and drives Th1 immune response. International Journal of Biological Macromolecules. 2017; 101: 398-407.

21. Ogura T, Hara H, Yokota S, Hosoe S, Kawase I, Kishimoto S, et al. Effector mechanism in concomitant immunity potentiated by intratumoral injection of Nocardia rubra cell wall skeleton. Cancer Research. 1985; 45: 6371-5.

22. Yamamura Y, Ogura T, Sakatani M, Hirao F, Kishimoto S, Fukuoka M, et al. Randomized controlled study of adjuvant immunotherapy with Nocardia rubra cell wall skeleton for inoperable lung cancer. Cancer Research. 1983; 43: 5575-9.

23. de Reijke TM, de Boer EC, Schamhart DH, Kurth KH. Immunostimulation in the urinary bladder by local application of Nocardia rubra cell wall skeleton preparation (Rubratin) for superficial bladder cancer immunotherapy--a phase I/II study. Urological Research. 1997; 25: 117-20. 
24. Routes JM, Morris K, Ellison MC, Ryan S. Macrophages kill human papillomavirus type 16 E6-expressing tumor cells by tumor necrosis factor alpha- and nitric oxide-dependent mechanisms. Journal of Virology. 2005; 79: 116-23.

25. Giampazolias E, Zunino B , Dhayade S, Bock F, Cloix C, Cao K, et al. Mitochondrial permeabilization engages NF- $\kappa$ B-dependent anti-tumour activity under caspase?deficiency[]]. Nature Cell Biology, 2017, 19(9):1116-29. 